\title{
Criminologie
}

\section{La police, un service municipal comme les autres}

\section{Henri-Paul Vignola}

Volume 17, numéro 1, 1984

La police après 1984

URI : https://id.erudit.org/iderudit/017194ar

DOI : https://doi.org/10.7202/017194ar

Aller au sommaire du numéro

Éditeur(s)

Les Presses de l'Université de Montréal

ISSN

0316-0041 (imprimé)

1492-1367 (numérique)

Découvrir la revue

Citer cet article

Vignola, H.-P. (1984). La police, un service municipal comme les autres.

Criminologie, 17(1), 127-131. https://doi.org/10.7202/017194ar d'utilisation que vous pouvez consulter en ligne.

https://apropos.erudit.org/fr/usagers/politique-dutilisation/ 
L'administration d'une municipalité comprend plusieurs facettes dont la sécurité publique. Le service de police, responsable de la paix et du bon ordre est, aux yeux des citoyens et des administrateurs municipaux, le service le plus important et à la fois le plus difficile à gérer efficacement.

Les raisons en sont nombreuses. D'abord, de multiples lois et de nombreuses procédures parfois complexes régissent ce métier. L'éventail des interventions auxquelles sont confrontés quotidiennement les policiers les amènent aussi bien à disposer sur le champ et à leur discrétion, des droits et des libertés de personnes qu'à régler des problèmes qui relèvent beaucoup plus des affaires sociales.

Le mythe autour du travail policier et plus spécifiquement celui des enquêteurs fait aussi montre de sa singularité. La ligne de démarcation entre la gestion des affaires courantes d'un service de police et la gestion des opérations policières est plutôt diffuse. Comme très peu d'administrateurs municipaux possèdent une expérience et une connaissance approfondie de la fonction policière, ils sont portés à $s$ 'en remettre aux dirigeants de la police persistant ainsi à entretenir le mystère autour de la fonction policière.

Dans la plupart des municipalités, un degré très large d'autonomie est accordée à la direction de la police, aussi bien pour la gestion des ressources humaines et physiques que pour celle des opérations policières. Une comparaison entre le service de police et les autres services dans plusieurs municipalités importantes permet de constater que les administrateurs municipaux n'accordent pas toujours à la police autant d'attention, de temps et d'énergie qu'ils le font pour les autres services sous leur gouverne.

Perçoivent-ils le service de police un peu comme un service «piégé» qu'il serait préférable de ne pas toucher pour éviter d'être confrontés à des problèmes' insoupçonnés? C'est parfois l'impression qu'ils laissent lorsqu'ils prétendent éviter de poser des questions pour ne pas se faire accuser d'ingérence. Sous ce fallacieux prétexte, les autorités de la ville négligent du même coup d'implanter dans le service de police les contrôles élémentaires et essentiels à une saine

* Commission des libérations conditionnelles, Ottawa. 
administration des affaires publiques. La Commission de police du Québec en arrive souvent à cette conclusion à la suite de ses enquêtes sur des corps policiers.

C'est vrai que la police, de par sa nature, est un domaine épineux et qu'elle suscite souvent des sentiments à la fois de frustration et d'inefficacité chez des administrateurs municipaux en plus de provoquer de la part des citoyens des réactions hostiles. Un incident tout à fait banal peut prendre des dimensions inattendues dans l'opinion publique. Prenons le cas de policiers qui auraient fait montre de force abusive contre des gens de couleur ou encore contre des mineurs. Le public et les médias vont conclure aussitôt que cette intervention est raciste et discriminatoire. Selon les proportions du geste et la publicité faite autour de cet incident, le risque sera grand de faire disparaitre subitement tous les efforts apportés au cours des dernières années par l'administration en place en vue d'améliorer les relations avec la communauté.

Une autre caractéristique propre à un service de police est le fait que tous les policiers à l'exception de son directeur et de quelques-uns de ses collaborateurs immédiats font tous partie de la même fraternité et d'une seule unité de négociation. Avec les années, ces fraternités de policiers sont devenues des syndicats actifs et militants dont les membres ont pris conscience de leur force d'impact quand vient le moment des négociations et du renouvellement des conventions collectives. Les officiers ou cadres intermédiaires, par le fait qu'ils sont intégrés avec leurs subalternes dans la même unité, viennent consolider encore plus la force du syndicat et son influence sur la gestion, sur les opérations policières et sur les services aux citoyens. Dans ces conditions, il en faut peu pour provoquer dans un service de police une crise de l'autorité et faire passer le leadership officiel de la direction de la police aux mains des dirigeants syndicaux.

Les administrateurs municipaux aussi bien élus que fonctionnaires, ont tous un défi à surmonter lorsqu'ils décident de travailler avec le service policier et ce, pour des raisons multiples. Un service de police est une organisation para-militaire avec port d'uniformes et de distinctions visibles des grades et du niveau d'autorité. Comme cette organisation formelle et hiérarchique s'inspire historiquement de l'armée, ses membres sont naturellement résistants aux changements organisationnels où des modèles flexibles d'administration seraient plus appropriés. 
La police est le service qui a la responsabilité d'appliquer le plus grand nombre de lois dans une ville en partant du code criminel, du code de la route et des divers règlements municipaux. Pour ce qui est de la réglementation municipale, les dirigeants municipaux devraient tenir compte de l'avis des policiers et discuter davantage avec eux des politiques d'application avant de sanctionner ou d'amender un règlement.

La police exerce aussi de vastes pouvoirs discrétionnaires pour une variété de décisions couvrant des circonstances de toute sorte. Les administrateurs au niveau de la gérance municipale auraient avantage à participer à la formation des policiers. Cette contribution dans la préparation des programmes de formation permettrait de donner une vue plus globale de l'administration municipale en collaborant à la formation de politiques et de procédures fondamentales susceptibles de modifier par la suite des attitudes erratiques ou des méthodes de travail inadaptées à l'époque que nous vivons.

Nous avons aussi observé que le directeur du service de police a des liens étroits avec la communauté, il est habituellement de la localité, il a fait carrière dans son service et il a été recruté des rangs à cause de la tradition, des pressions vénant du milieu ou encore à cause de la non-transférabilité du fonds de pension. Le poste de directeur de police en est un prestigieux où le titulaire est perçu comme une personnalité notoire et importante disposant de la sympathie et de l'appui des organismes sociaux, des associations d'hommes d'affaires et aussi de la majorité des citoyens.

La police est le plus visible des services de la municipalité. Les autos de patrouille sillonnent constamment les rues de la cité pour répondre à une large variété d'appels d'assistance allant des accidents de la circulation, des enfants perdus, des chats dans les arbres aux victimes de crimes. Les citoyens voient les agents de police au cours de leur patrouille de routine plus souvent que tous les autres employés municipaux ensemble.

La criminalité est un autre élément du défi à surmonter. Elle crée des situations de tension chez la population qui subit parfois un état de panique à forte saveur émotive sous l'influence de l'opinion publique.

Comme nous vivons dans une société démocratique, les dirigeants policiers doivent être sensibles à toute demande légitime de service. Toutefois, à cause des ressources limitées et des restrictions budgétaires, il est parfois nécessaire d'établir un ordre de priorité 
dans les services à dispenser aux citoyens. Cependant, déterminer ces priorités opérationnelles ainsi que la nature et le niveau de service ne devrait pas être le fardeau exclusif des dirigeants policiers. Cette décision concerne au premier plan les citoyens et, par délégation, les élus municipaux parce que ce sont eux qui contrôlent les moyens financiers et ce sont eux qui doivent répondre finalement aux contribuables de la bonne gestion de leur mandat. La police, dans une démocratie, demeure subordonnée à l'autorité constituée par la loi et incarnée dans la personne des élus et, par délégation, dans la gérance municipale. C'est pourquoi la police doit être un service public au service des citoyens et qui doit s'adapter à la réalité contemporaine de son milieu.

Bien qu'une partie du travail de la police soit de caractère confidentiel, un service de police en tant qu'organisme public doit prendre toutes les mesures pour renseigner les citoyens sur sa gestion et pour rendre accessible l'information d'intérêt public. Il est à l'avantage des dirigeants policiers de faire montre de transparence. La meilleure façon d'obtenir l'appui des citoyens et des autorités en place, c'est de faire la démonstration d'une administration policière progressive, proche des citoyens, et ouverte à l'innovation i.e. une administration prête à se remettre en question et à accepter des idées nouvelles.

Un climat propice et serein est essentiel aux bonnes relations entre l'administration municipale et les dirigeants policiers. Les policiers pour être efficaces doivent sentir qu'ils peuvent compter sur un appui honnête et soutenu de la part des autorités en place. Il sera alors facile aux administrateurs municipaux et policiers d'évaluer ensemble les besoins de la municipalité en sécurité publique et d'arriver à établir les objectifs du service de police. Par la suite, le directeur de police sera en mesure de soumettre un plan d'action en vue de la réalisation de ces objectifs, plan qui pourra être soumis aux membres du conseil municipal dans le cadre de la présentation du budget annuel.

Les administrateurs municipaux doivent se rendre de plus en plus à l'évidence: la population est soucieuse et inquiète de sa sécurité et la police est trop importante pour ne recevoir qu'une attention passive ou indifférente. Pour les autorités municipales, la police est le défi le plus difficile mais aussi le plus stimulant. Les enjeux sont trop grands pour risquer un échec. L'administration municipale a une responsabilité sociale grave et pour accomplir convenablement son mandat, elle doit exercer un leadership efficace et rigoureux. Les 
conditions primordiales de succès pour les autorités locales consistent à travailler étroitement et en équipe avec les responsables de la police, à considérer ce service en priorité à cause de la nature de son travail et à établir des relations harmonieuses entre la police et les autres organismes relevant de l'administration de la justice ou ayant un rapport avec la sécurité publique.

Une phrase célèbre de Clémenceau lui faisait dire que la guerre était trop importante pour être confiée à des militaires. De même, nous pourrions affirmer que «la police est trop importante pour être confiée à des policiers». Les administrateurs municipaux ont non seulement le droit mais l'obligation de voir à ce que leur communauté reçoive de la police un service juste, efficace et conforme aux normes d'une société démocratique. C'est la seule façon pour la police de demeurer un service municipal comme les autres. 